\title{
A Study Of The ST Changes In The aVR Lead On 12-Lead ECG To Identify Infarct - Related Artery (IRA) In Patients With Acute Inferior Wall Myocardial Infarction
}

\author{
Aditya Mahaseth' ${ }^{1}$, Bikas Nepal', Biplave Karki', Jeet Ghimire' ${ }^{1}$, Naveen Pandey ${ }^{1}$, Prashant Shah', Prahlad Karki' \\ ${ }^{1}$ Department of Cardiology, BPKIHS, Dharan, Nepal
}

\begin{abstract}
BACKGROUND

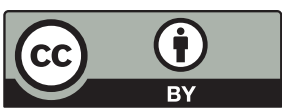

This work is licensed under a Creative Commons Attribution 4.0 Unported License.

Lead avR is a valuable but mostly ignored lead in clinical electrocardiography. Recently, ST-segment depression in lead aVR during an inferior wall myocardial infarction has been suggested as a predictor of LCX artery involvement.
\end{abstract}

\section{METHODS}

This study was a single centre cross sectional observational study done in BPKIHS, Dharan from February 2018 to January 2020. Patients presenting to the OPD or emergency room of BPKIHS diagnosed as acute inferior wall myocardial infarction based on clinical symptoms, ECG and/or Cardiac tropinin I levels, and planned for coronary angiography, meeting the inclusion and exclusion criterias were included.

\section{RESULTS}

Among 134 cases, male:female ratio was 1.3:1. Overall, 38 patients (28.4\%) were found to have aVR depression and 96 patients (71.6\%) were without aVR depression. The culprit artery was found to be the right coronary artery in 95 patients (70.9\%), the LCX in 39 patients (29.1\%). The sensitivity and specificity of ST-segment depression in lead aVR for LCX as the culprit artery were $92.3 \%$ and $97.9 \%$ respectively. Positive predictive and Negative predictive value for LCX as the culprit arteries were $94.74 \%$ and $96.87 \%$. The sensitivity, specificity, positive predictive value and negative predictive value for RCA as the culprit artery were $97.89 \%, 92.3 \%, 96.89 \%$ and $94.73 \%$ respectively.

\section{CONCLUSION}

Significant ST depression in aVR is associated with a higher specificity and good sensitivity for LCX lesions, the ST changes in this lead should be carefully examined in all patients who are suspected of having inferior wall myocardial infarction.

\section{BACKGROUND}

Lead avR is a valuable but mostly ignored lead in clinical electrocardiography ${ }^{(1)}$. Standard 12-lead electrocardiography (ECG) is a widely available technology that is routinely applied in the setting of chest pain to identify patients with ST-elevation myocardial infarction (STEMI) who would benefit from emergent revascularization. ${ }^{(2)}$

The use of ECG to predict the location of the culprit coronary lesion within the infarct-related artery (IRA) could provide additional valuable information to augment clinical decision making and expedite reperfusion therapy. Previous studies in patients with acute coronary syndromes have correlated ECG findings to the results of coronary angiography, leading to the formulation of ECG criteria capable of identifying the coronary artery housing the culprit lesion and the site of the culprit lesion within that artery. The culprit artery of anterior STEMI is nearly always the left anterior descending artery (LAD), but inferior STEMI can be caused by an occlusion of either the right coronary artery (RCA) or left circumflex (LCX) artery. Recently the conventional ECG findings used to predict culprit artery in inferior STEMI were less useful in patients with dominant LCX infarction. Few studies had correlated aVR depression with the relative dominance of the RCA and LCX.(3)

Inferior wall involvement in myocardial infarction is a common clinical scenario, and can be due to involvement of either the right coronary artery or the left circumflex artery. Traditional criterias to predict the infarct related artery have been historically present but none of them are 100 percent specific. Recently ST depression in lead aVR has been postulated to be a predictor of LCX artery involvement. So this study was undertaken to determine whether ST

\footnotetext{
*Corresponding Author I

Dr. Aditya Mahaseth, MD, DM Cardiology

Department of Cardiology, BPKIHS, Dharan, Nepal

Email: amahaseth@hotmail.com
} 
segment depression in lead aVR predicts left circumflex artery as the infarct related artery in acute inferior wall myocardial infarction cases.

\section{METHODOLOGY}

This study was a single centre cross sectional observational study done in BPKIHS, Dharan from February 2018 to January 2020. Patients presenting to the OPD or emergency room of BPKIHS diagnosed as acute inferior wall myocardial infarction based on clinical symptoms, ECG and/or Cardiac tropinin I levels, and planned for coronary angiography, meeting the inclusion and exclusion criterias were included.

Inclusion Criteria - Patients who meet the following criteria were included in the study:

1) typical anginal chest pain lasting $30 \mathrm{~min}$ before hospital admission;

2) ST elevation $0.1 \mathrm{mV}$ in at least 2 of the 3 inferior leads (II, III, and aVF);

3) ST deviation in aVR;

4) elevation of the creatine kinase and its MB fraction to greater than twice of the upper limit and or positive tropinin I;

5) undergoing coronary angiography during hospitalization, where infarct related artery confirmed.

Exclusion Criteria - Patients with the following conditions were excluded from the study:

1) With a previous history of acute myocardial infarction, coronary artery bypass surgery or percutaneous coronary intervention prior to the current hospitalization;

2) ECG evidence of bundle branch block or left ventricular hypertrophy; and

3) Significant stenosis (more than 70\%) in both RCA and LCX so that a single IRA could not be defined.

Data Collection - Patients presenting to the OPD or emergency room of BPKIHS diagnosed as acute inferior wall myocardial infarction based on clinical symptoms, ECG and/or Cardiac tropinin I levels, and planned for coronary angiography, meeting the inclusion and exclusion criterias were included in the study. A detailed socio-demographic data was collected and the information recorded in structured Performa. The demographic characteristics included age, sex, and detail signs and symptoms, and clinical parameters. Chest radiograph, ECG and ECHO findings were recorded from the opd and ward of BPKIHS as deemed necessary. The angiographic data was correlated with the clinical and ECG finding and data was entered into
SPSS for statistical analysis. The ecg and angiographic finding was confirmed by two certified cardiologists.

Coronary Angiography - Coronary angiography was performed during hospitalization in all patients. The coronary angiography films were reviewed by investigators who were certified cardiologist. The IRA was identified by either

1) a total occlusion or a significant stenosis (70\%) of the RCA or LCX, or their major branches;

2) arteriographic evidence of an intraluminal thrombus.

Electrocardiographic analysis - The 12 lead ECGs recorded on admission of all patients was analyzed by the investigator. In each of the 12 standard leads, ST segment deviation from the isoelectric line was measured 0.06 seconds after the $J$ point. Measurement was made to the nearest $0.25 \mathrm{~mm}$. The TP segment was used as the isoelectric line unless tachycardia had caused fusion of the $T$ and $P$ waves, in which case the PR segment was used.

SAMPLE SIZE (N): This was a descriptive cross sectional study. Maskey et al. have reported the prevalence of coronary artery disease to be $5 \%$ and Abhinav vaidya et al. 2011 at 6\%. Adhikari et al. reported the incidence of inferior wall $\mathrm{MI}$ to be $35.9 \%$ amongst the STEMI cases admitted to SGNHC, Kathmandu.

Sample size was calculated on the basis of specificity of $96 \%$ and prevalence of $80 \%$ to be 245 based on the following formula

Sample size $(n)$ based on sensitivity $=\frac{Z_{1-\alpha / 2}^{2} \times S_{N} \times\left(1-S_{N}\right)}{L^{2} \times \text { Prevalence }}$, and

sample size $(n)$ based on specificity $=\frac{Z_{1-\alpha / 2}^{2} \times S_{p} \times\left(1-S_{p}\right)}{L^{2} \times(1-\text { Prevalence })}$,

$\mathrm{N}=$ sample size, $\mathrm{Z}=\mathrm{Z}$ score $=1.96$ at $95 \%$ confident interval, $L=$ margin of error $=5 \%$ at $95 \%$ confidence interval.

However since this was a hospital based study the corrected sample size based upon the annual incidence of acute inferior wall MI cases in BPKIHS.

$\mathrm{CSS}=\mathrm{N} /(1+\mathrm{n} / \mathrm{N})$

The calculated sample size was One hundred and twenty one (121 denominator of study) patients meeting the case Definition of Acute inferior wall myocardial infarction were enrolled in study. Sample size was taken based upon previous records of hospital admission of AIWMI, published literature to conduct such studies and for the reason of convenience and practicality. 
Statistical analysis - Collected data was entered in Microsoft excel 2007 and uploaded into SPSS 17 version. For descriptive statistics percentage, mean, standard deviation, median, IQR (Interquartile range) was calculated and along with graphical and tabular presentation.

For inferential statistics Chi-square test and Fishers exact test were used to compare differences in discrete variables at $95 \%$ confidence interval. $P$ value $<0.05$ will be considered as significant. Pearson coefficient correlation analysis will be done to establish a correlation among the different quantitative variables, such as the ST deviation in different leads, between the LCX and RCA groups. Multivariate logistic regression analysis was performed to assess the predictive value of ST depression in lead aVR in LCX-caused inferior infarction. $P$ value less than 0.05 was considered to be statistically significant.

Written consent was taken from the patient party.

I declare no conflict of interest.

\section{RESULTS}

\section{General Findings}

A total of 134 patients (male 76 and female 58) were included in the study, aged 35 to 87 years (mean age 60.84 $\pm 11)$.
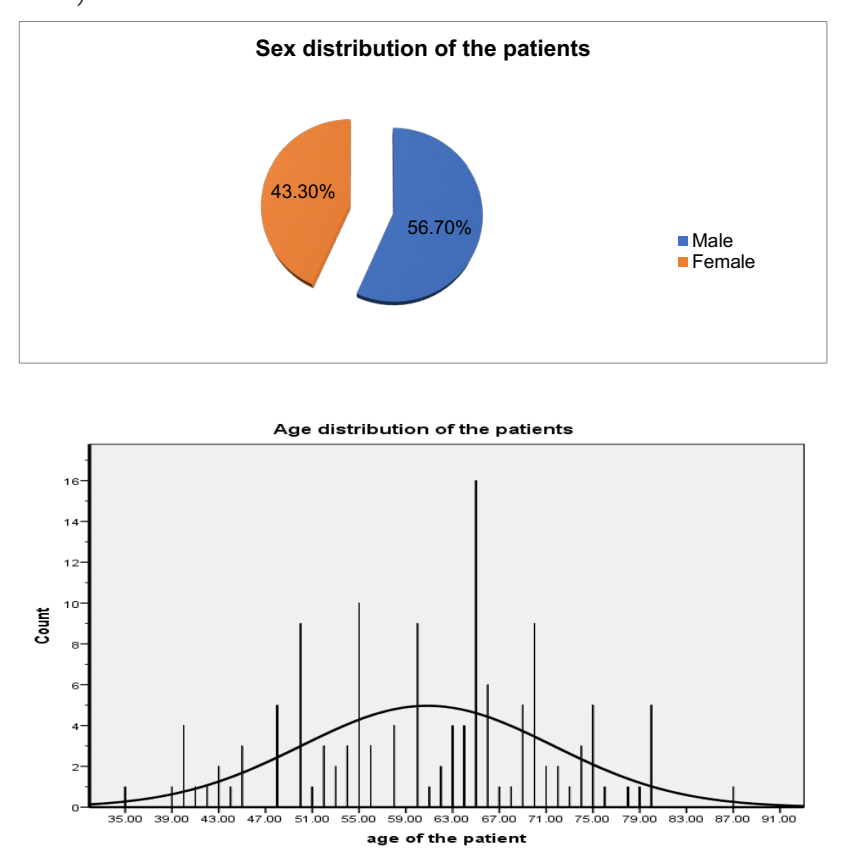

\section{Risk Factors}

1) Diabetes Mellitus

Out of the total 134 patients 37 patients had diabetes mellitus while 97 patients did not have diabetes.

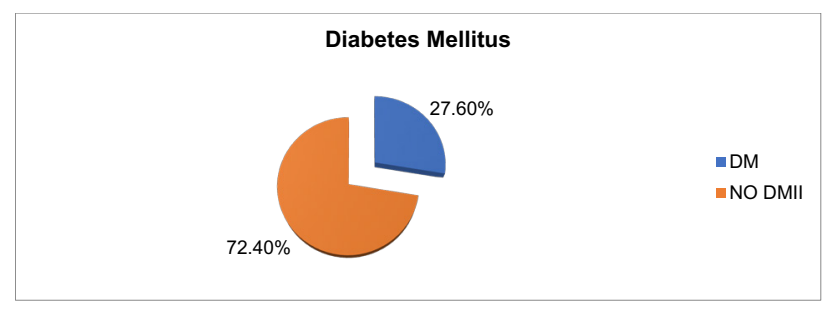

2) Hypertension

Hypertension was a major risk factor among the patients presenting with inferior wall STEMI. 57 patients had hypertension based on history or hospital records. 77 patients did not have hypertension.

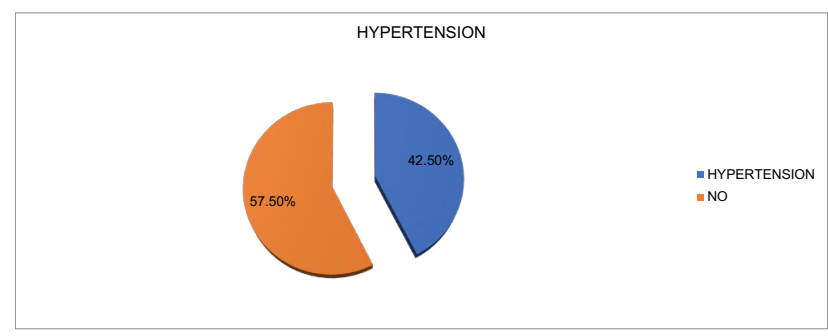

3) Smoking

72 patients did not have a history of smoking or tobacco use. Out of the rest, 48 were currently active smokers and 14 were reformed smokers.

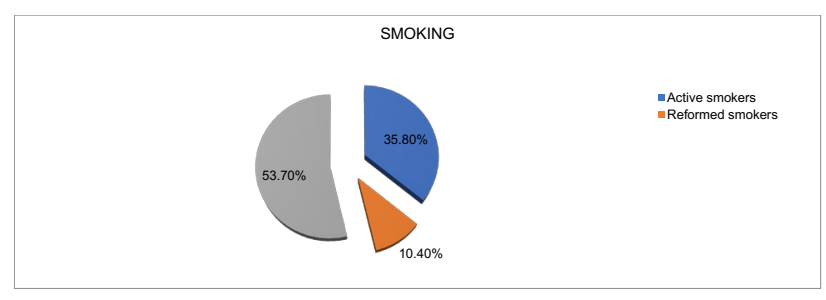

4)

Dyslipidemia

16 patients had dyslipidemia based on laboratory tests or history of treatment for dyslipidemia.

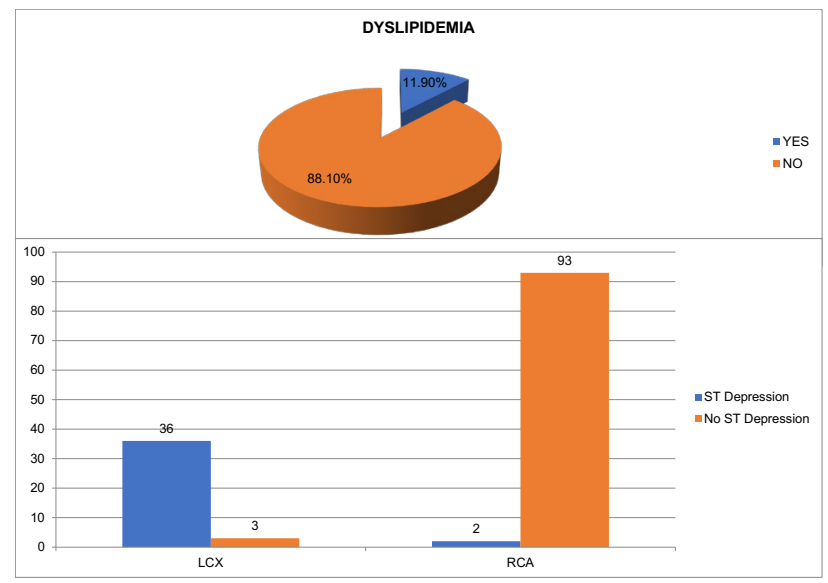

The LCX disease was found significantly higher proportion in ST depression in lead aVR (n-36; 94.74\%) than without ST depression in lead aVR $(n-3 ; 3.2 \%) \quad(p<0.0001)$. The 
sensitivity and specificity of ST-segment depression in lead aVR for LCx as the culprit artery were $92.3 \%$ and $97.9 \%$ respectively. Positive predictive and Negative predictive value for LCx as the culprit arteries were $94.74 \%$ and $96.87 \%$.

However, 96 patients, with ST segment elevation or STsegment isoelectric or minimal ST segment depression in the lead aVR, classified as patients without depression in lead aVR were significant for RCA as the culprit artery in 93 $(96.87 \%)$ compared to $2(5.26 \%)$ ( $p$ 0001) patients. The sensitivity,

specificity, positive predictive value and negative predictive value for RCA as the culprit artery were 97.89\%, 92.3\%, $96.89 \%$ and $94.73 \%$ respectively.

\section{ST Depression and culprit artery localization}

\begin{tabular}{|c|c|c|c|c|c|}
\hline \multirow[b]{2}{*}{$\mathrm{Ag}$} & \multirow[b]{2}{*}{$\mathrm{Fr})$} & \multirow[b]{2}{*}{ Per } & \multicolumn{2}{|c|}{ culprit artery } & \multirow{2}{*}{ Total } \\
\hline & & & Icx & rca & \\
\hline \multirow{5}{*}{$\begin{array}{l}\text { lead } \\
\text { avr st } \\
\text { segment }\end{array}$} & \multirow{3}{*}{$\begin{array}{l}\text { No ST } \\
\text { depres- } \\
\text { sion }\end{array}$} & & & & \\
\hline & & Count & 3 & 93 & 96 \\
\hline & & $\begin{array}{l}\% \text { within } \\
\text { culprit }\end{array}$ & $7.7 \%$ & $97.9 \%$ & $71.6 \%$ \\
\hline & \multirow[t]{2}{*}{$\begin{array}{l}\text { ST de- } \\
\text { pression }\end{array}$} & Count & 36 & 2 & 38 \\
\hline & & $\begin{array}{l}\% \text { within } \\
\text { culprit } \\
\text { artery }\end{array}$ & $92.3 \%$ & $2.1 \%$ & $28.4 \%$ \\
\hline \multirow[t]{2}{*}{ Total } & \multirow{2}{*}{$\begin{array}{l}\text { Count } \\
\% \text { within } \\
\text { culprit } \\
\text { artery }\end{array}$} & 17.1 & 39 & 95 & 134 \\
\hline & & 100.0 & $100.0 \%$ & $100.0 \%$ & $100.0 \%$ \\
\hline
\end{tabular}

Prognostic differences between anterior and inferior wall Myocardial Infarction (MI) has been extensively investigated, but there is limited information about similar comparison between inferior wall $\mathrm{Ml}$ caused by right coronary artery (RCA) and left circumflex artery (LCX) occlusion. In a study LCX artery involvement has been associated with greater MR occurrence, lower LVEF values and poor outcome. ${ }^{(5)}$

Therefore, patients with ECG finding in favour of LCX occlusion should be considered as high risk and an invasive approach should be planned.

\section{Other Findings}

The average window period of presentation to the hospital was 27.06 ( \pm 27 ) hours, which highlights the fact that most of the patients in our country present $t$ the hospital late. In a study done by Mishra et al. ${ }^{(6)}$ in India it was shown that less than half $(47.77 \%)$ presented within the window period of 12 hours while only $0.8 \%$ of patients availed the golden period of 1 hour.

However the average window period of presentation in female group was 25.22 hours, while in males was 29.46 hours. This finding echoed the result of previous studies in which there were no sex based differences in diagnostic performance in first medical contact. ${ }^{(7)}$

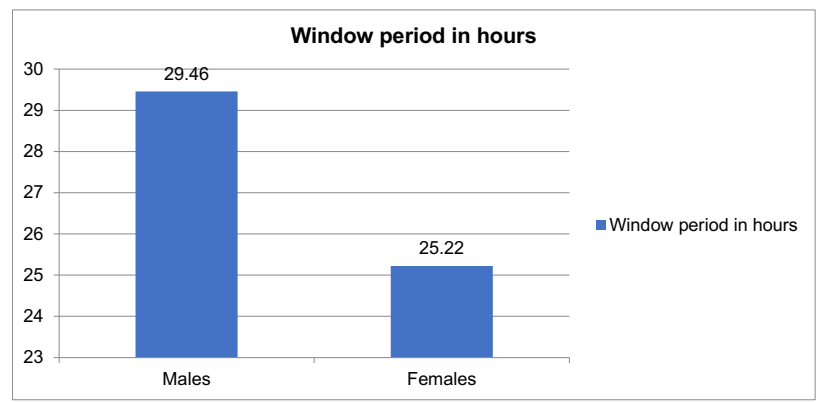

Among the patients presenting within the window period 13 patients underwent systemic thrombolysis, amongst which there were 1 instance of failed thrombolysis.

5 Patients underwent primary balloon angioplasty and 23 patients underwent stent implantation in the 1st hospital stay.

The average LV ejection fraction of the patients presenting with acute inferior wall STEMI was $49.29 \pm 7.15$.

Eight patients developed complete heart block which recovered in all but 1 . There were total two deaths one in the RCA group and one in the LCx group. Two patients developed ventricular tachycardia which subsided with drug treatment. Six patients developed Acute Kidney Injury during hospital stay.

There was no correlation of the degree of ST Segment depression in lead avR to KILLIP Class, Complications or Death/Recovery, although it would be expected that greater degree of ST segment depression will suggest a greater myocardial tissue at jeopardy, a hypothesis which needs validation.

\section{Subgroup Analysis}

115 patients were in KILLIP Class I at presentation out of which $72.2 \%$ had RCA as the culprit artery, 6 and 3 patients were in KILLIP Class II and III respectively. There were 10 patients who were KILLIP Class IV on presentation out of which $50 \%$ had RCA and the other $50 \%$ had LCX as the culprit artery. There were more KIILIP Class I patients in the RCA subgroup.

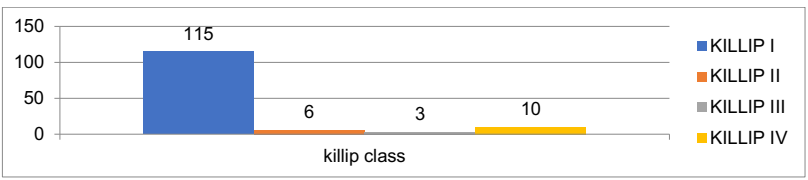




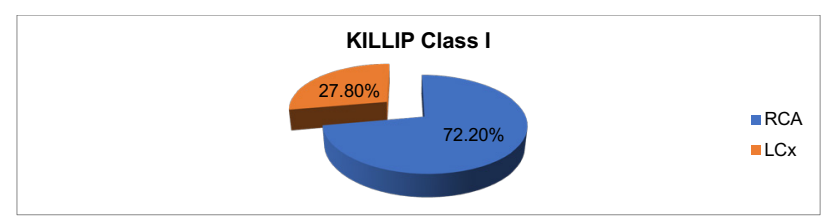

\section{Discussion}

The ECG changes during LCX artery occlusion is highly variable because of its anatomical distribution. The LCX supplies blood to the posterior, posterolateral, posteroinferior, or posteroinferolateral myocardium.(8) During LCX occlusion, posterolateral wall involvement is associated with proximal occlusion because this region is supplied, at least in part, by the obtuse marginal branch of the LCX artery. When occlusion of the LCX artery more distally, the posterolateral wall will not be involved, and the ST-segment vector will be oriented more inferiorly.(8)

In this study there were total of 134 patients out of which 76 were males and 58 were females. In 2013, the prevalence of $\mathrm{Ml}$ in men was about three times higher than for women in the UK.(11) Raitt et al(12) documented a more marked ST segment elevation among women in the case of STEMI located anteriorly, although the differences were not statistically significant. Pelter et al(13) documented the greater, although not significantly so, ST elevation in men during the performance of angioplasty in anterior descending and circumflex coronary arteries, although it was not observed when right coronary artery was involved.

The present study found that in patients with inferior myocardial infarction, the RCA is much more likely than the LCX to be the culprit artery, with a ratio of 2.44: 1. These results are consistent with previous studies by other groups

where the RCA to LCX ratio was found to vary from $2.2: 1$ to $7.0: 1 .^{(9)}$

In most patients the myocardial distribution of the RCA is slightly rightward in the frontal plane, and consequently the current of injury resulting from its occlusion will be reflected more in lead III than in lead II. Conversely, the distribution of the LCX is slightly leftward in the frontal plane, and the current of injury from its closure will be seen more in lead II than in lead III. An injury vector leftward enough to cause STsegment elevation in lead I is common with LCX occlusion, but rare with $\mathrm{RCA}$ occlusion.

The current of injury with RCA occlusion is more or less perpendicular to the axis of lead aVR, whereas the current of injury resulting from occlusion of the LCX has a mean vector that forms an obtuse angle with the axis of aVR. Therefore, significant ST-segment depression in aVR is more likely to occur with LCX occlusion, as shown in the present study. ST segment in lead V1 may be elevated when RCA is occluded because it is influenced by the current of injury from right ventricular infarction. ST-segment depression both in V1 and $\mathrm{V} 2$ is the reciprocal change resulting from STsegment elevation in posterior wall of the left ventricle and is typical of LCX occlusion.(14)

The $\mathrm{aVL}$ lead faces the high-lateral segment of the left ventricle, and is the onlylead truly reciprocal to the inferior wall. Inferior-wall myocardial infarction caused by RCA occlusion has greater STsegment depression in lead aVL than in lead I.(15)

The present study demonstrated that ST-segment depression in lead aVR is a good indicator for LCX occlusion, with a sensitivity of $92.3 \%$ and a specificity of $97.9 \%$. aVR is frequently ignored during the analysis of inferior myocardial infarction, but some investigators have suggested that this lead can provide information useful for the characterization of inferior myocardial infarction.(16)

Kosuge and coworkers(17) studied inferior myocardial infarction with inferior ST-segment elevation in which TIMI grade 3 flow of the RCA or LCX was achieved within 6 hours from symptom onset. They found that the degree of ST- segment depression in lead aVR is a useful predictor of impaired myocardial reperfusion in patients who have experienced inferior myocardial infarction; a significant ST depression in lead aVR is useful for identifying patients who are most likely to benefit from aggressive therapeutic strategies designed to improve myocardial reperfusion.(17)

Our study found only a small proportion of the patients with RCA-related myocardial infarction has a ST depression in aVR. However, 94.74\% of patients with LCX-related inferior myocardial infarction had a ST depression of $0.1 \mathrm{mV}$ or more in aVR. The specificity and sensitivity of using aVR criteria in diagnosing LCX involvement is $92.3 \%$ and $97.9 \%$, respectively. These results indicate that use of a combination of traditional ECG criteria plus aVR ST depression may enhance the accuracy of 12-lead ECG in differentiating the culprit artery in patients with inferior-wall myocardial infarction.

\section{Limitations Of The Study}

This was a small sample size, prospective study in a single center. The study didn't deal in predicting the lesion either proximal or distal coronary occlusion, mega artery involvement. The study lacked the follow-up and complications like reinfarction, post infarction angina, arrhythmias and mortality.

The electrocardiographic findings in this study might differ considerably from those patients with previous episodes of myocardial infarction, late presentation. Thus, the findings 
cannot be generalized to all patients with inferior wall AMI and large-scale studies are needed to confirm the present findings. I do not think that using these ECG criteria should replace angiography, but it would provide earlier identification of the culprit artery.

Another limitation of the present study is that the role of V3-V6 and V3R, V4R in the differential diagnosis was not evaluated and compared with aVR.

There have been reports showing that in patients with LCXrelated acute inferior myocardial infarction, there is a higher frequency of ST elevation in V4-V6. ${ }^{(18)}$

In LCX-related inferior myocardial infarction, the amount of ST depression is often greater than the amount of ST elevation in V3. ST elevation in V3R and V4R is compelling evidence of RCA involvement and the presence of right ventricular infarction. ${ }^{(19)}$

\section{CONCLUSION}

1) Significant ST depression in aVR is associated with a higher specificity and good sensitivity for LCX lesions, the ST changes in this lead should be carefully examined in all patients who are suspected of having inferior wall myocardial infarction.

2) Cardiologists should pay more attention to the tracing of lead aVR when interpreting the 12-lead ECG in clinical practice.

\section{REFERENCES}

1. Gorgels APM, Engelen DJM, Wellens HJJ. Lead aVR, a mostly ignored but very valuable lead in clinical electrocardiography. Journal of the American College of Cardiology. 2001;38(5):13556.

2. Yamaji $H$, Iwasaki $K$, Kusachi $S$, Murakami T, Hirami R, Hamamoto $\mathrm{H}$, et al. Prediction of acute left main coronary artery obstruction by 12-lead electrocardiography. ST segment elevation in lead aVR with less ST segment elevation in lead V(1). J Am Coll Cardiol. 2001;38(5):1348-54.

3. Mahmoud K, M. Abd Al Rahman T, Taha H, Mostafa S. Significance of ST-segment deviation in lead aVR for prediction of culprit artery and infarct size in acute inferior wall STelevation myocardial infarction. The Egyptian Heart Journal. 2014;67(2):145-9.

4. Menown IBA, Adgey AAJ. Improving the ECG classification of inferior and lateral myocardial infarction by inversion of lead aVR. Heart. 2000;83(6):657-60.

5. Sohrabi B, Separham A, Madadi R, Toufan M, Mohammadi $\mathrm{N}$, Aslanabadi N, et al. Difference between Outcome of Left Circumflex Artery and Right Coronary Artery Related Acute Inferior Wall Myocardial Infarction in Patients Undergoing Adjunctive Angioplasty after Fibrinolysis. J Cardiovasc Thorac Res. 2014;6(2):101-4.
6. Mishra TK, Das B. ST-Segment Elevated Acute Myocardial Infarction: Changing Profile Over Last 24 Years. The Journal of the Association of Physicians of India. 2016;64(6):28-34.

7. Sörensen NA, Neumann JT, Ojeda F, Schäfer S, Magnussen C, Keller T, et al. Relations of Sex to Diagnosis and Outcomes in Acute Coronary Syndrome. Journal of the American Heart Association [Internet]. 2018 2018/03//; 7(6).

8. Chia BL, Yip JW, Tan HC, Lim YT. Usefulness of ST elevation II/III ratio and ST deviation in lead I for identifying the culprit artery in inferior wall acute myocardial infarction. The American journal of cardiology. 2000;86(3):341-3.

9. Zimetbaum PJ, Krishnan S, Gold A, Carrozza JP, 2nd, Josephson ME. Usefulness of ST-segment elevation in lead III exceeding that of lead II for identifying the location of the totally occluded coronary artery in inferior wall myocardial infarction. The American journal of cardiology. 1998;81(7):918-9.

10. Barnhill JE, 3rd, Tendera M, Cade H, Campbell WB, Smith RF. Depolarization changes early in the course of myocardial infarction: significance of changes in the terminal portion of the QRS complex. J Am Coll Cardiol. 1989;14(1):143-9.

11. Bhatnagar $P$, Wickramasinghe $K$, Williams J, Rayner $M$, Townsend $\mathrm{N}$. The epidemiology of cardiovascular disease in the UK 2014. Heart. 2015;101(15):1182-9.

12. Raitt MH, Litwin PF, Martin JS, Weaver WD. ECG findings in acute myocardial infarction. Are there sex-related differences? Journal of electrocardiology. 1995;28(1):13-6.

13. Pelter MM, Adams MG, Drew BJ. ST segment deviation during myocardial ischemia: are there gender differences? Progress in cardiovascular nursing. 1996;11(3):4-9.

14. Mak KH, Chia BL, Tan AT, Johan A. Simultaneous ST-segment elevation in lead V1 and depression in lead V2. A discordant ECG pattern indicating right ventricular infarction. Journal of electrocardiology. 1994;27(3):203-7.

15. Herz I, Assali AR, Adler Y, Solodky A, Sclarovsky S. New electrocardiographic criteria for predicting either the right or left circumflex artery as the culprit coronary artery in inferior wall acute myocardial infarction. The American journal of cardiology. 1997;80(10):1343-5.

16. Gorgels AP, Engelen DJ, Wellens HJ. Lead aVR, a mostly ignored but very valuable lead in clinical electrocardiography. J Am Coll Cardiol. 2001;38(5):1355-6.

17. Kosuge M, Kimura K, Ishikawa T, Ebina T, Hibi K, Toda N, et al. ST-segment depression in lead aVR: a useful predictor of impaired myocardial reperfusion in patients with inferior acute myocardial infarction. Chest. 2005;128(2):780-6.

18. Kontos MC, Desai PV, Jesse RL, Ornato JP. Usefulness of the admission electrocardiogram for identifying the infarct-related artery in inferior wall acute myocardial infarction. The American journal of cardiology. 1997;79(2):182-4.

19. Birnbaum Y, Drew BJ. The electrocardiogram in ST elevation acute myocardial infarction: correlation with coronary anatomy and prognosis. Postgrad Med J. 2003;79(935):490-504. 